\title{
Sport and second chances?
}

\section{All drug cheats should be banned for life, here's why.}

\author{
Philippe Crisp and Jamie Sims \\ The University of Chichester
}

\begin{abstract}
The purpose of this critical commentary is to highlight the inconsistencies evident within the discourse of Performance Enhancing Drug (PED) use and Anti-Doping violations. Of most note, the issue related to proper rehabilitation and subsequent reintegration of athletes who have failed drugs tests is reliant on a notion that when athletes return to competition, fairness will prevail. We know that PEDS, in particular steroids and exogenous hormone treatment, confer an advantage even without concurrent training (see Bhasin et.al. 1996). That their effectiveness is not in doubt is consistent with current policy. However, the question of just how advantageous it is for athletes to use them, even just the once, and whether there are any permanent advantages to doing so, is not particularly evident in contemporary discourse. This paper takes the position, using emerging scientific evidence as well as the recollections of UK strength sports administrators, that any consideration of 'clean' sport needs to resolve policy with the evidence that permanent advantages accrued from PED use can only be combatted by promoting a 'natural for life' standard.
\end{abstract}

Keywords: performance enhancing drugs; anti-doping; tactical drug use; zero tolerance; natural.

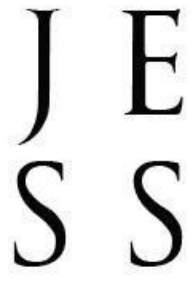

\section{Critical Commentary}

Journal of Emerging Sport Studies

Volume 2, (2019): London, Ontario

All Rights Reserved, 2019: ISSN - 2562-3184 
Currently, the World Anti-Doping Agency's (WADA) Anti-Doping Rule Violations (ADRVs) allow a sliding scale of transgressions to take place with no lifetime bans given in the first instance of a failed test. However, this perpetuates a system that ultimately does not distinguish between those who have never used Performance Enhancing Drugs (PEDs) and those who have. There is, however, and as we will outline, a very real distinction between these two categories. This is particularly so when the nature of the PEDs moves away from the more temporary acting examples (for instance, stimulants and diuretics) to exogenous hormones administered to increase performance (such as anabolic steroids and growth hormones). Discourse regarding this issue remains typically undeveloped, at best so marginal, as to have not entered any real debate concerning the morality of drug use within sport. What discourse there is inevitably relies far more on the principles of how to deal with those who have failed a test once or even several tests (for instance Justin Gatlin in the Olympics, and Jon Jones in the Ultimate Fighting Championship [UFC]), and how they can be both rehabilitated and reintroduced into mainstream sport. We therefore argue that any consideration of how to test, what to test for, how to penalise failed tests, and whether sport should allow drug use (e.g. see Savalescu et al, 2004) needs to be reframed. This is especially relevant given that the prevailing argument related to the use of PEDs within sport and subsequent reintegration lacks a considered engagement with key scientific research.

A seemingly perpetual succession of drug scandals within sport over the last 40 years illustrates that individual athletes, training camps, coaches, and even state sponsored federations (perhaps most notably the former East Germany in the 1960s and 70s) encouraged and facilitated a culture of systematic PED use. Yet despite efforts, every decade since the 1980s, with Ben Johnson's positive test at the 1988 Seoul Olympics of particular note, has seen major drug scandals in sport. Some of the more notable cases were the Irish swimmer Michelle Collins in the 1990s, the Bay Area Laboratory Co-Operative (BALCO) scandal (that ultimately led to the fall of Marion Jones and Tom Montgomery) in the early 2000s, Lance Armstrong's eventual lifetime ban from the Union Cycliste Internationale (UCI) in 2012 (that initiated soul searching within cycling from the 2000 s to the present), and the continuing issues of state sponsored programmes (such as Russia) within the 2010 s.

More recently, the major sports leagues in the USA continue to be beset by repeated instances of PED use that seem to appear to suggest a permissive culture. Alongside this, the continued monitoring of some state sport systems, such as Russia, is still beset with problems. Now there are increased calls to examine what seems to be the incredibly high prevalence of athletes with medical conditions that require what some, perhaps cynically, but perhaps entirely pragmatically, state might be fortuitously timed episodes of medicinal (with acknowledged performance enhancing qualities) intervention that fall under the banner of Therapeutic Use Exemption (TUE). Examples include episodes initially exposed by the cyber-espionage group the Russian Fancy Bears who released documents related to multiple British sport stars and include the incidents regarding the British cyclists Bradley Wiggins and Chris Froome. Added to this there is now what seems to be a partial redistribution of medals and places from the 2008 and 2012 Olympic Games thanks to developments in testing procedures and the retesting of older samples. It is no surprise then that public opinion of professional and elite sport being rife with PEDs is palpable. 
In truth, some believe that it is difficult for any part of life to be completely drug free at this point in contemporary postindustrial society, with an argument that drugs (whether legal, recreational, illegal, or PEDs) are completely integrated and normalised into everyday life and that to separate athletes from this reality would be impossible. Indeed, the notion of drug free sport is further questioned by many who believe that there are a range of factors that cloud issues of morality, whilst at the same time promoting how regulated drug use could reduce what we already know regarding the dangers of drug use in sport (Coakley and Pike, 2009). The founding premise adopted here then, is that a system which monitors athlete risk and mitigates potential damage to health through medical intervention is one that is more realistic and more attuned to the realities of modern life.

This manner of concession then, one explicitly or implicitly stating that the battle against PEDS is futile, suggests that given contemporary prevalence and sophistication, testing is now redundant (Savalescu et al, 2004). This outlook appears to shift the use of PEDs in sport to a more permissive system of explicitly monitoring athlete health against known risk factors rather than the current preventative system (Schneider \& Butcher, 2000). The argument for allowing PED use also stretches into one that asks questions of how excellence in non-sport fields is facilitated. Savalescu et al. (2004), for instance, highlight the fact that other performance type 'art' allows or encourages recreational drugs to enhance creativity; that the 'spirit' of sport would be enhanced by allowing genetic manipulation; that allowing drugs would permit an even playing field; and that permitted drug use would be safer overall.

In response to this line of argument Devine (2010) reasoned against permitting PEDs because it would unsettle the 'balance of excellences' in sport (e.g. power vs skill) with a consequential reduction in audience enjoyment. Devine (2010) also raises the moral objection against PEDs in that sport is defined as an activity in which participants agree to adhere to the rules. Here, Devine (2010) draws upon the classic work of Suits (1978), who stated that the very essence of games and sports was that the participants would adhere to the rules. This definition helps establish what can be considered a clear distinction of what sport 'is', i.e. an activity that fundamentally places the idea of mutually agreed guidelines and typically inefficient means to complete goals, as the centrepiece. Within this wider debate Kayser and Smith (2008) argue for greater leniency, citing the rising cost-benefit of drug testing, while Pope et al. (2014) urge caution against this approach given current ignorance surrounding long-term adverse consequences of PED use, including the withdrawal symptoms associated with suppressed hypothalamic-pituitary-testicular (HPT) function and mental health conditions.

Whilst some argue that the majority of top class athletes use PEDs (thus in their eyes obviating the need to monitor lower level sport) and that, as mentioned previously, drug use is commonplace in modern society, that all drugs carry risks, and that these risks should prove to be the burden of individuals, testing for PEDs within sport does still take place. This appetite for drug testing remains with WADA, and other organisations, to police the use of PEDs in sports. We argue that it is essential to continue this policing yet also call for a wider discussion regarding the use of PEDS because any argument either for leniency or for specific periods of ban does not consider the long-lasting effects of PED use. These effects are, in no 
uncertain terms, advantages conferred to even one-time users of PEDS such that athletes continue to benefit post use. As emerging evidence shows, these advantages may be on a permanent basis, rendering any PEDs transgression ultimately worthwhile if natural barriers to excellence have been broken. This therefore either removes the option of winning for clean athletes, perhaps even at amateur level, or forces them to take PEDs in order to maintain any sense of parity with those who do or, and central to our argument, ever have.

There are two fundamental elements underpinning our position. The first argument is relatively simple, in that any idea of creating a level playing field by allowing PEDs neglects to take into account what are very simple medicinal understandings of reactions to all drugs - with, for instance, common and uncommon reactions (again, simple medicinal terminology) and side effects to drugs by definition acknowledging that individuals react differently to the same drugs and/or dosages. Extending this understanding to the context of PEDs, this means that there can be a wide variation in the way that individuals may experience both performance enhancing and negative side-effects. Put simply, someone might well just have a fantastic genetic predisposition to respond very positively to certain types of PEDs, which, as we currently understand it, is not considered a talent (admittedly at the moment). Under a more permissive system, then, sporting success may partially rely on a genetic predisposition to optimal PED response.

The second argument is perhaps more complicated, but also represents the main thrust of this article. A reframing of the argument for allowing drugs and indeed even for allowing athletes that have failed drugs tests and served bans before coming back, is necessary. This is due to emerging evidence suggesting that using certain PEDs (e.g. exogenous anabolic steroids) results in permanent morphological changes, which subsequently confer lifetime advantages to any PED user in comparison with a 'clean' athlete. If this is so, the debate needs to be opened up far more explicitly and critically at this point and to encompass the conflicting tension between allowing for rehabilitation of offenders and explicitly recognising that doing so facilitates a continuing disparity in terms of fairness.

We acknowledge that the evidence in this respect is far from complete. Some is suppositional, extrapolating upon the work undertaken by Egner et al. (2013) on mammals. In their study, Egner et al. (2013) subjected female mice to episodes of either a) an experimental group experiencing testosterone exposure (through a pellet inserted in the skin of their necks) for 14 days, or b) a control group experiencing placebo pellets with no testosterone. They found that the number of myonuclei in the group subjected to testosterone therapy increased by $66 \%$, with an increase also in fibre cross-sectional fibre area (CSA) of $77 \%$. Importantly, while fibre size for this group decreased to baseline levels after three weeks, the increase in myonuclei was observed at a three-month follow-up. On reintroduction of overload-exercise, fibre CSA areas grew by $31 \%$ while controls grew only $6 \%$. This increase in myonuclei persisted long after the administration of the drug, strongly indicating permanent change. Clearly, any extrapolation to humans must accept that mammalian function and response to cellular memory follow similar pathways (Allen et al., 1995; Gunderson, 2016). In the context of human physical performance, therefore, the advantages conferred by additional myonuclei in sport would be enhanced muscle contractile activity and strength, and increased capacity for hypertrophy. These are significant components that underpin sports performance, increasing 
concerns that PED use in humans will demonstrate results, potentially including permanently increased muscle myonuclei.

Indeed, there already is some evidence that suggests this is the case. Using a small sample of competitive powerlifters, Eriksson (2006) studied current PEDs users $(n=9)$, those that had never used PEDs $(n=10)$, and those that had previously used PEDs $(n=7)$. Biopsies from participants trapezius and vastus lateralis muscles indicated increased fibre areas and myonuclei, as well as increased central nuclei, within both those who were currently using and those who had previously used PEDs, concluding that "some of the morphological changes induced by testosterone and anabolic steroids are very long lasting, perhaps lifelong" (p. 42).

Moreover, Crisp's research, (2016a; 2016b), tracing the history of strength sports in the United Kingdom, revealed what can be considered a rife and unchecked PEDs culture at the time. Exploring the recollections and stories of some of the key administrators of strength sports in the UK reveals their attempt to create 'lifelong' natural strength movements to counter this PEDs culture. This approach to creating lifetime drug-free lifting federations was underpinned by a variety of examples from the participants (the key administrators) in the study. For example, all of them recollected seeing many people improve from regional to near international status in very short periods of time and improving approximately $20 \%$ after having had static performances for a decade or more. They also reported beliefs that any accrued improvements were also, once the PEDs were discontinued, still above the previously 'naturally' held limit that the users had reached. These recollections then support the findings of the scientific community through observations of PED users and the effects, when 'on' and 'off' drugs over a period of 30+ years, strongly endorsing the message that there are permanent effects of drug use beyond a single episode of consumption.

While the issues of long-term advantages of PED use within humans remains inadequately studied, Egner et al. (2013) and Gunderson (2016) have already called for a reconsideration of continued athlete eligibility in the context of failed tests. As it stands then, enough evidence exists to at least call into question any argument related to the use of drugs within sport that allows subsequent rehabilitation of offenders. There is also sufficient evidence to suggest that drug cheats are prospering from the permanent advantages that even a single episode of drug use confers. Furthermore, this raises the spectre of tactical drug use early in athletes' careers whereby the prospect of being caught is insufficiently punitive. Or, having been caught, the prospect that long term benefits will continue into their later careers.

Consequently, we argue that if we are to develop the debate on the policing of PED use, then an informed and evidence-based understanding of the exact consequences PEDs imbue, regarding permanently breaking natural barriers to excellence, must be established. This is especially so when considering an ethical and moral viewpoint, as at present this information is not being taken into account in these debates and judgements. In short, some may need to acknowledge that any move away from performance, doping, and elitism, to values of fairness and equity, requires a clean for life movement with zero tolerance of PED use. 


\section{References}

Allen, D. L., Monke, S. R., Talmadge, R. J., Roy, R. R. and Edgerton, V. R. (1995) Plasticity of myonuclear number in hypertrophied and atrophied mammalian skeletal muscle fibers, Journal of Applied Physiology, 78, 1969-1976.

Bhasin, S., Storer, t., Berman, N., Callegari, C., Levenger, B., Phillips, J., Bunnell, T., Tricker, R., Shirazi, A. and Casaburi, R. (1996) The Effects of Supraphysiologic Doses of Testosterone on Muscle Size and Strength in Normal Men, The New England Journal of Medicine, 335 (1), 1-7.

Coakley, J. and Pike, E. (2009) Sports in Society: Issues and Controversies, Berkshire, McGraw Hill.

Crisp, P. (2016a) All-Round Weightlifting: Small Spaces, Big Weights - the Recreation and Reconceptualisation of a Strength Sport. In C, Howley and S. Dun (eds) The Playing Field Making sense of Spaces and Places in Sporting Culture, Oxfordshire, Inter-Disciplinary Press, 157-166.

Crisp (2016b) The Line in the Sand for British Strength Sports: The Creation of a Drug Free for Life Ethos, ID. Net - Probing the Boundaries - Sport 5 Global Conference, September, Oxford.

Devine, W.J. (2010) Doping is a threat to sporting excellence, British Journal of Sports Medicine, published online June 11, 2010

Egner, I., Bruusgard, J.C., Eftestøl, E., and Gundersen, K. (2013) A cellular memory mechanism aids overload hypertrophy in muscle long after an episodic exposure to anabolic steroids, The Journal of Physiology, 15(591,Pt 24), 6221-6230.

Erikkson, A. (2006) Strength Training and Anabolic Steroids: A comparative study of the vastus lateralis, a thigh muscle and the trapezius, a shoulder muscle, of strength-trained athletes. UMEA UNIVERSITY MEDICAL DISSERTATIONS.

Gundersen, K. (2016) Muscle memory and a new cellular model for muscle atrophy and Hypertrophy, Journal of Experimental Biology, 219, 235-242.

Kayser, B. and Smith, A.C.T. (2008) Globalisation of anti-doping: the reverse side of the medal, British Medical Journal, 337, 85-87

Pope, H. G. Jr., Wood, R. Rogol, A., Nyberg, F, Bowers, L. and Bhasin, S. (2014) Adverse Health Consequences of Performance-Enhancing Drugs: An Endocrine Society Scientific

Statement, Endocrine Reviews, 35(3), 341-375. 
Sport and Second Chances? - Crisp and Sims

Savulescu, J., Foddy,B., and Clayton, M. (2004) Why we should allow performance enhancing drugs in sport, British Journal of Sports Medicine, 38, 666-670,

Schneider, A. and Butcher, R. (2000) A philosophical overview of the arguments on banning doping in sport. In T. Tannsjo and C. Tamburrini (eds) Values in Sport: Elitism, nationalism, gender equality and the scientific manufacture of winners, London, E\&FN Spor, 185-199.

Suits, B. (1978) The Grasshopper: Games, Life and Utopia, London, University of Toronto Press. 